\title{
Arcwise connectedness of the solution set of a nonclosed nonconvex integral inclusion
}

\author{
Aurelian Cernea
}




\title{
ARCWISE CONNECTEDNESS OF THE SOLUTION SET OF A NONCLOSED NONCONVEX INTEGRAL INCLUSION
}

\author{
AURELIAN CERNEA
}

Received 21 November, 2006

\begin{abstract}
We consider a class of nonconvex nonclosed integral inclusions and we prove the arcwise connectedness of the solution set of this problem.
\end{abstract}

2000 Mathematics Subject Classification: 34 A60

Keywords: integral inclusion, set-valued contraction, fixed point

\section{INTRODUCTION}

This note is concerned with the following Fredholm type integral inclusion

$$
\begin{gathered}
x(t)=\lambda(t)+\int_{0}^{1} f(t, s, u(s)) d s, \\
u(t) \in F(t, x(t), G(t, x(t))) \quad \text { a.e. in } I,
\end{gathered}
$$

where $X$ is a separable Banach space, $I:=[0,1], F: I \times X \times X \rightarrow \mathcal{P}(X), G: I \times X \rightarrow$ $\mathcal{P}(X), \lambda: I \rightarrow X$, and $f: I \times I \times X \rightarrow X$.

When $F$ does not depend on the last variable, (1.2) reduces to

$$
u(t) \in F(t, x(t)) .
$$

In this case, existence results for problem (1.1), (1.3) and the stability of the solution set with respect to small perturbations of the free term are obtained via fixed point techniques in Theorem 2.8.9 in [6] (see also references therein). In [6], the set-valued map $F$ is assumed to be at least closed-valued. Such an assumption is quite natural in order to obtain good properties of the solution set, but it is also interesting to investigate the problem when the right-hand side of (1.3) may have nonclosed values.

Following the approach in [4,5], we consider the problem (1.1), (1.2), where $F$ and $G$ are closed-valued set-valued maps, Lipschitzian with respect to the second variable and $F$ is contractive in the third variable. Obviously, the right-hand side of the inclusion in (1.2) is in general neither convex nor closed. We prove the arcwise connectedness of the solution set to (1.1), (1.2). The main tool is a result $[4,5]$ 
concerning the arcwise connectedness of the fixed point set of a class of nonconvex nonclosed set-valued contractions.

This idea was already used for similar results for other classes of differential inclusions [1-4]. We note that a similar result for Volterra type integral inclusions is obtained in [1], but it is well known that, in general, the results for Fredholm integral equations cannot be obtained from the corresponding results for Volterra integral equations.

The paper is organized as follows: in Section 2, we recall some preliminary results that we use in the sequel and, in Section 3, we prove our main result.

\section{PReliminaries}

Let $Z$ be a metric space with the distance $d_{Z}$ and let $\mathcal{P}(Z)$ be the family of all nonempty closed subsets of $Z$. For $a \in Z$ and $A, B \in \mathcal{P}(Z)$, set $d_{Z}(a, B)=$ $\inf _{b \in B} d_{Z}(a, b)$ and $d_{Z}^{*}(A, B)=\sup _{a \in A} d_{Z}(a, B)$. Denote by $D_{Z}$ the PompeiuHausdorff generalized metric on $\mathcal{P}(Z)$ defined by

$$
D_{Z}(A, B)=\max \left\{d_{Z}^{*}(A, B), d_{Z}^{*}(B, A)\right\}, \quad A, B \in \mathcal{P}(Z) .
$$

In what follows, when the product $Z=Z_{1} \times Z_{2}$ of metric spaces $Z_{i}, i=1,2$, is considered, it is assumed that $Z$ is equipped with the distance

$$
d_{Z}\left(\left(z_{1}, z_{2}\right),\left(z_{1}^{\prime}, z_{2}^{\prime}\right)\right)=\sum_{i=1}^{2} d_{Z_{i}}\left(z_{i}, z_{i}^{\prime}\right) .
$$

Let $X$ be a nonempty set and let $F: X \rightarrow \mathcal{P}(Z)$ be a set-valued map from $X$ to $Z$. The range of $F$ is the set $F(X)=\bigcup_{x \in X} F(x)$. Let $(X, \mathcal{F})$ be a measurable space. The set-valued map $F: X \rightarrow \mathcal{P}(Z)$ is called measurable if $F^{-1}(\Omega) \in \mathscr{F}$ for any open set $\Omega \subset Z$, where $F^{-1}(\Omega)=\{x \in X ; F(x) \cap \Omega \neq \varnothing\}$. Let $\left(X, d_{X}\right)$ be a metric space. The set-valued map $F$ is called Hausdorff continuous if, for any $x_{0} \in X$ and every $\varepsilon>0$, there exists $\delta>0$ such that $x \in X, d_{X}\left(x, x_{0}\right)<\delta$ implies $D_{Z}\left(F(x), F\left(x_{0}\right)\right)<\varepsilon$.

Let $(T, \mathcal{F}, \mu)$ be a finite, positive, nonatomic measure space and let $\left(X,|\cdot|_{X}\right)$ be a Banach space. We denote by $L^{1}(T, X)$ the Banach space of all (equivalent classes of) Bochner integrable functions $u: T \rightarrow X$ endowed with the norm

$$
\|u\|_{1}=\int_{T}|u(t)|_{X} d \mu .
$$

A nonempty set $K \subset L^{1}(T, X)$ is called decomposable if, for every $u, v \in K$ and every $A \in \mathcal{F}$, one has

$$
\chi_{A} \cdot u+\chi_{T \backslash A} \cdot v \in K
$$

where $\chi_{B}$ indicates the characteristic function of $B \in \mathcal{F}$.

A metric space $Z$ is called an absolute retract if, for any metric space $X$ and any nonempty closed set $X_{0} \subset X$, every continuous function $g: X_{0} \rightarrow Z$ has a continuous 
extension $g: X \rightarrow Z$ over $X$. It is obvious that every continuous image of an absolute retract is an arcwise connected space.

In what follows we recall some preliminary results that are the main tools in the proof of our result.

Let $(T, \mathcal{F}, \mu)$ be a finite, positive, nonatomic measure space, $S$ a separable Banach space and let $\left(X,|\cdot|_{X}\right)$ be a real Banach space. To simplify the notation we write $E$ in place of $L^{1}(T, X)$.

Lemma 1 ([4]). Assume that $\varphi: S \times E \rightarrow \mathcal{P}(E)$ and $\psi: S \times E \times E \rightarrow \mathcal{P}(E)$ are Hausdorff continuous set-valued maps with nonempty, closed, decomposable values, satisfying the following conditions:

(a) There exists $L \in[0,1)$ such that, for every $s \in S$ and every $u, u^{\prime} \in E$,

$$
D_{E}\left(\varphi(s, u), \varphi\left(s, u^{\prime}\right)\right) \leq L\left\|u-u^{\prime}\right\|_{E} .
$$

(b) There exists $M \in[0,1)$ such that $L+M<1$ and for every $s \in S$ and every $(u, v),\left(u^{\prime}, v^{\prime}\right) \in E \times E$,

$$
D_{E}\left(\psi(s, u, v), \psi\left(s, u^{\prime}, v^{\prime}\right)\right) \leq M\left(\left\|u-u^{\prime}\right\|_{E}+\left\|v-v^{\prime}\right\|_{E}\right) .
$$

Set $\operatorname{Fix}(\Gamma(s, \cdot))=\{u \in E ; u \in \Gamma(s, u)\}$, where $\Gamma(s, u)=\psi(s, u, \varphi(s, u)),(s, u) \in$ $S \times E$. Then

(1) For every $s \in S$ the set $\operatorname{Fix}(\Gamma(s, \cdot))$ is nonempty and arcwise connected.

(2) For any $s_{i} \in S$ and any $u_{i} \in \operatorname{Fix}(\Gamma(s, \cdot)), i=1, \ldots, p$, there exists a continuous function $\gamma: S \rightarrow E$ such that $\gamma(s) \in \operatorname{Fix}(\Gamma(s, \cdot))$ for all $s \in S$ and $\gamma\left(s_{i}\right)=u_{i}, i=1, \ldots, p$.

Lemma 2 ([4]). Let $U: T \rightarrow \mathcal{P}(X)$ and $V: T \times X \rightarrow \mathcal{P}(X)$ be two nonempty closed-valued set-valued maps satisfying the following conditions:

(a) $U$ is measurable and there exists $r \in L^{1}(T, \mathbb{R})$ such that $D_{X}(U(t),\{0\}) \leq r(t)$ for almost all $t \in T$.

(b) The set-valued map $t \mapsto V(t, x)$ is measurable for every $x \in X$.

(c) The set-valued map $x \mapsto V(t, x)$ is Hausdorff continuous for all $t \in T$.

Let $v: T \rightarrow X$ be a measurable selection from $t \mapsto V(t, U(t))$.

Then there exists a selection $u \in L^{1}(T, X)$ such that $v(t) \in V(t, u(t)), t \in T$.

Let $X$ be a separable Banach space, $C(I, X)$ the Banach space of continuous functions defined on $I$ with values in $X$ endowed with the norm $\|x\|_{C}=\sup _{t \in I}|x(t)|$. We consider the problem (1.1), (1.2) defined by the mappings $F: I \times X \times X \rightarrow \mathcal{P}(X)$, $G: I \times X \rightarrow \mathcal{P}(X), \lambda: I \rightarrow X$, and $f: I \times I \times X \rightarrow X$. A function $x \in C(I, X)$ is said to be a solution of (1.1), (1.2) if there exists a function $u \in L^{1}(I, X)$ such that (1.1), (1.2) are satisfied.

In order to study problem (1.1), (1.2) we introduce the following hypothesis. 
Hypothesis 1. Let $\lambda \in C(I, X)$ and $F: I \times X \times X \rightarrow \mathcal{P}(X), G: I \times X \rightarrow \mathcal{P}(X)$ be two set-valued maps with nonempty closed values, satisfying the following assumptions:

(i) The set-valued maps $t \mapsto F(t, u, v)$ and $t \mapsto G(t, u)$ are measurable for all $u, v \in X$.

(ii) There exist $l \in L^{1}(I, \mathbb{R})$ such that, for arbitrary $u, u^{\prime} \in X$,

$$
D\left(G(t, u), G\left(t, u^{\prime}\right)\right) \leq l(t)\left|u-u^{\prime}\right| \text { a.e. in } I .
$$

(iii) There exist $m \in L^{1}(I, \mathbb{R})$ and $\theta \in[0,1)$ such that, for arbitrary $u, v, u^{\prime}, v^{\prime} \in$ $X$,

$$
D\left(F(t, u, v), F\left(t, u^{\prime}, v^{\prime}\right)\right) \leq m(t)\left|u-u^{\prime}\right|+\theta\left|v-v^{\prime}\right| \text { a.e. in } I .
$$

(iv) There exist $g_{1}, g_{2} \in L^{1}(I, \mathbb{R})$ such that

$$
d(\{0\}, F(t,\{0\},\{0\})) \leq g_{1}(t), \quad d(\{0\}, G(t,\{0\})) \leq g_{2}(t) \text { a.e. in } I .
$$

(v) $f: I \times I \times X \rightarrow X$ is a continuous and bounded function and there exists a constant $M>0$ such that

$$
\left|f\left(t, s, u_{1}\right)-f\left(t, s, u_{2}\right)\right| \leq M\left|u_{1}-u_{2}\right| \quad \forall u_{1}, u_{2} \in X .
$$

For $\lambda \in C(I, X)$, we denote by $S(\lambda)$ the solution set of (1.1), (1.2). In what follows, $N(t)=\max \{l(t), m(t)\}, t \in I$, and $N^{*}=\int_{0}^{1} N(s) d s$.

\section{THE MAIN RESULT}

Even if the set-valued map from the right-hand side of (1.1), (1.2) has, in general, nonclosed nonconvex values, the solution set $S(\lambda)$ has some meaningful properties, stated in Theorem 1 below.

Theorem 1. Assume that Hypothesis 1 is satisfied and $2 N^{*} M+\theta<1$. Then:

(1) For every $\lambda \in C(I, X)$, the solution set $S(\lambda)$ of (1.1), (1.2) is nonempty and arcwise connected in the space $C(I, X)$.

(2) For any $\lambda_{i} \in C(I, X)$ and any $x_{i} \in S\left(\lambda_{i}\right), i=1, \ldots, p$, there exists a continuous function $s: C(I, X) \rightarrow C(I, X)$ such that $s(\lambda) \in S(\lambda)$ for any $\lambda \in C(I, X)$ and $s\left(\lambda_{i}\right)=x_{i}, i=1, \ldots, p$.

(3) The set $S=\cup_{\lambda \in C(I, X)} S(\lambda)$ is arcwise connected in $C(I, X)$.

Proof. (1) For $\lambda \in C(I, X)$ and $u \in L^{1}(I, X)$, set

$$
x_{\lambda}(t)=\lambda(t)+\int_{0}^{1} f(t, s, u(s)) d s, \quad t \in I .
$$

We shall prove that the set-valued maps $\varphi: C(I, X) \times L^{1}(I, X) \rightarrow \mathcal{P}\left(L^{1}(I, X)\right)$ and $\psi: C(I, X) \times L^{1}(I, X) \times L^{1}(I, X) \rightarrow \mathcal{P}\left(L^{1}(I, X)\right)$, given by the formulas

$$
\varphi(\lambda, u)=\left\{v \in L^{1}(I, X) ; v(t) \in G\left(t, x_{\lambda}(t)\right) \text { a. e. in } I\right\}
$$


and

$$
\psi(\lambda, u, v)=\left\{w \in L^{1}(I, X) ; w(t) \in F\left(t, x_{\lambda}(t), v(t)\right) \text { a. e. in } I\right\}
$$

for $\lambda \in C(I, X)$ and $u, v \in L^{1}(I, X)$, satisfy the hypotheses of Lemma 1 .

Since $x_{\lambda}$ is measurable and $G$ satisfies Hypothesis 1(i) and (ii), the set-valued map $t \mapsto G\left(t, x_{\lambda}(t)\right)$ is measurable and nonempty closed-valued, it has a measurable selection. Therefore, due to Hypothesis $1(\mathrm{iv})$, the set $\varphi(\lambda, u)$ is nonempty. The fact that the set $\varphi(\lambda, u)$ is closed and decomposable follows by simple computation. In the same way we obtain that $\psi(\lambda, u, v)$ is a nonempty closed decomposable set.

Pick $(\lambda, u),\left(\lambda_{1}, u_{1}\right) \in C(I, X) \times L^{1}(I, X)$ and choose $v \in \varphi(\lambda, u)$. For each $\varepsilon>0$ there exists $v_{1} \in \varphi\left(\lambda_{1}, u_{1}\right)$ such that, for every $t \in I$, one has

$$
\begin{aligned}
\left|v(t)-v_{1}(t)\right| & \leq D\left(G\left(t, x_{\lambda}(t)\right), G\left(t, u_{\lambda_{1}}(t)\right)\right)+\varepsilon \\
& \leq N(t)\left(\left|\lambda(t)-\lambda_{1}(t)\right|+\int_{0}^{1} M\left|u(s)-u_{1}(s)\right| d s\right)+\varepsilon .
\end{aligned}
$$

Hence

$$
\left\|v-v_{1}\right\|_{1} \leq N^{*}\left\|\lambda-\lambda_{1}\right\|_{C}+N^{*} M\left\|u-u_{1}\right\|_{1}+\varepsilon
$$

for any $\varepsilon>0$.

This implies

$$
d_{L^{1}(I, X)}\left(v, \varphi\left(\lambda_{1}, u_{1}\right)\right) \leq N^{*}\left\|\lambda-\lambda_{1}\right\|_{C}+N^{*} M\left\|u-u_{1}\right\|_{1}
$$

for all $v \in \varphi(\lambda, u)$. Therefore,

$$
d_{L^{1}(I, X)}^{*}\left(\varphi(\lambda, u), \varphi\left(\lambda_{1}, u_{1}\right)\right) \leq N^{*}\left\|\lambda-\lambda_{1}\right\|_{C}+N^{*} M\left\|u-u_{1}\right\|_{1} .
$$

Consequently,

$$
D_{L^{1}(I, X)}\left(\varphi(\lambda, u), \varphi\left(\lambda_{1}, u_{1}\right)\right) \leq N^{*}\left\|\lambda-\lambda_{1}\right\|_{C}+N^{*} M\left\|u-u_{1}\right\|_{1},
$$

which shows that $\varphi$ is Hausdorff continuous and satisfies the assumptions of Lemma 1.

Let us pick $(\lambda, u, v),\left(\lambda_{1}, u_{1}, v_{1}\right) \in C(I, X) \times L^{1}(I, X) \times L^{1}(I, X)$ and choose $w \in$ $\psi(\lambda, u, v)$. Then, as before, for each $\varepsilon>0$ there exists $w_{1} \in \psi\left(\lambda_{1}, u_{1}, v_{1}\right)$ such that

$$
\begin{aligned}
\mid w(t)- & w_{1}(t) \mid \leq D\left(F\left(t, x_{\lambda}(t), v(t)\right), F\left(t, x_{\lambda_{1}}(t), v_{1}(t)\right) b i g\right)+\varepsilon \\
& \leq N(t)\left(\left|\lambda(t)-\lambda_{1}(t)\right|+M \int_{0}^{1}\left|u(s)-u_{1}(s)\right| d s\right)+\theta\left|v(t)-v_{1}(t)\right|+\varepsilon
\end{aligned}
$$

for every $t \in I$. Hence

$$
\begin{aligned}
\left\|w-w_{1}\right\|_{1} & \leq N^{*}\left\|\lambda-\lambda_{1}\right\|_{C}+N^{*} M\left\|u-u_{1}\right\|_{1}+\theta\left\|v-v_{1}\right\|_{1}+\varepsilon \\
& \leq N^{*}\left\|\lambda-\lambda_{1}\right\|_{C}+\left(N^{*} M+\theta\right)\left(\left\|u-u_{1}\right\|_{1}+\left\|v-v_{1}\right\|_{1}\right)+\varepsilon \\
& \leq N^{*}\left\|\lambda-\lambda_{1}\right\|_{C}+\left(N^{*} M+\theta\right) d_{L^{1}(I, X) \times L^{1}(I, X)}\left((u, v),\left(u_{1}, v_{1}\right)\right)+\varepsilon .
\end{aligned}
$$


As above, we deduce that

$$
\begin{aligned}
& D_{L^{1}(I, X)}\left(\psi(\lambda, u, v), \psi\left(\lambda_{1}, u_{1}, v_{1}\right)\right) \\
& \quad \leq N^{*}\left\|\lambda-\lambda_{1}\right\|_{C}+\left(N^{*} M+\theta\right) d_{L^{1}(I, X) \times L^{1}(I, X)}(u, v),\left(u_{1}, v_{1}\right),
\end{aligned}
$$

namely, the set-valued map $\psi$ is Hausdorff continuous and satisfies the hypothesis of Lemma 1.

Let us put $\Gamma(\lambda, u)=\psi(\lambda, u, \varphi(\lambda, u)),(\lambda, u) \in C(I, X) \times L^{1}(I, X)$. According to Lemma 1, the set $\operatorname{Fix}(\Gamma(s, \cdot))=\{u \in E ; u \in \Gamma(s, u)\}$ is nonempty and arcwise connected in $L^{1}(I, X)$. Moreover, for fixed $\lambda_{i} \in C(I, X)$ and $u_{i} \in \operatorname{Fix}\left(\Gamma\left(\lambda_{i}, \cdot\right)\right)$, $i=1, \ldots, p$, there exists a continuous function $\gamma: C(I, X) \rightarrow L^{1}(I, X)$ such that

$$
\gamma(\lambda) \in \operatorname{Fix}(\Gamma(\lambda, \cdot)), \quad \forall \lambda \in C(I, X)
$$

and

We shall prove that

$$
\gamma\left(\lambda_{i}\right)=u_{i}, \quad i=1, \ldots, p
$$

$$
\operatorname{Fix}(\Gamma(\lambda, \cdot))=\left\{u \in L^{1}(I, X) ; u(t) \in F\left(t, x_{\lambda}(t), G\left(t, x_{\lambda}(t)\right)\right) \text { a. e. in } I\right\} .
$$

Denote by $A(\lambda)$ the right-hand side of (3.3). If $u \in \operatorname{Fix}(\Gamma(\lambda, \cdot))$, then there is $v \in$ $\varphi(\lambda, v)$ such that $u \in \psi(\lambda, u, v)$. Therefore, $v(t) \in G\left(t, x_{\lambda}(t)\right)$ and

$$
u(t) \in F\left(t, x_{\lambda}(t), v(t)\right) \subset F\left(t, u_{\lambda}(t), G\left(t, u_{\lambda}(t)\right)\right) \quad \text { a. e. in } I,
$$

so that $\operatorname{Fix}(\Gamma(\lambda, \cdot)) \subset A(\lambda)$.

Let now $u \in A(\lambda)$. By Lemma 2, there exists a selection $v \in L^{1}(I, X)$ of the set-valued map $\left.t \rightarrow G\left(t, x_{\lambda}(t)\right)\right)$ satisfying

$$
u(t) \in F\left(t, x_{\lambda}(t), v(t)\right) \quad \text { a.e. in } I .
$$

Hence, $v \in \varphi(\lambda, v), u \in \psi(\lambda, u, v)$ and thus $u \in \Gamma(\lambda, u)$, which completes the proof of (3.3).

We next note that, taking into account Hypothesis $1(\mathrm{v})$, the function $T: L^{1}(I, X) \rightarrow$ $C(I, X)$,

$$
T(u)(t):=\int_{0}^{1} f(t, s, u(s)) d s, \quad t \in I,
$$

is continuous and one has

$$
S(\lambda)=\lambda+T(\operatorname{Fix}(\Gamma(\lambda, \cdot))), \quad \lambda \in C(I, X) .
$$

Since $\operatorname{Fix}(\Gamma(\lambda, \cdot))$ is nonempty and arcwise connected in $L^{1}(I, X)$, the set $S(\lambda)$ has the same properties in $C(I, X)$.

(2) Let $\lambda_{i} \in C(I, X)$ and let $x_{i} \in S\left(\lambda_{i}\right), i=1, \ldots, p$ be fixed. By (3.4), there exists $v_{i} \in \operatorname{Fix}\left(\Gamma\left(\lambda_{i}, \cdot\right)\right)$ such that

$$
x_{i}=\lambda_{i}+T\left(v_{i}\right), \quad i=1, \ldots, p .
$$


If $\gamma: C(I, X) \rightarrow L^{1}(I, X)$ is a continuous function satisfying (3.1) and (3.2), we define, for every $\lambda \in C(I, X)$,

$$
s(\lambda)=\lambda+T(\gamma(\lambda)) .
$$

Obviously, the function $s: C(I, X) \rightarrow C(I, X)$ is continuous, $s(\lambda) \in S(\lambda)$ for all $\lambda \in$ $C(I, X)$, and

$$
s\left(\lambda_{i}\right)=\lambda_{i}+T\left(\gamma\left(\lambda_{i}\right)\right)=\lambda_{i}+T\left(v_{i}\right)=x_{i}, \quad i=1, \ldots, p .
$$

(3) Let $x_{1}, x_{2} \in S=\cup_{\lambda \in C(I, X)} S(\lambda)$ and choose $\lambda_{i} \in C(I, X), i=1,2$ such that $x_{i} \in S\left(\lambda_{i}\right), i=1$, 2. From the conclusion of (2) we deduce the existence of a continuous function $s: C(I, X) \rightarrow C(I, X)$ satisfying $s\left(\lambda_{i}\right)=x_{i}, i=1,2$ and $s(\lambda) \in S(\lambda)$, $\lambda \in C(I, X)$. Let $h:[0,1] \rightarrow C(I, X)$ be a continuous mapping such that $h(0)=\lambda_{1}$ and $h(1)=\lambda_{2}$. Then the function $s \circ h:[0,1] \rightarrow C(I, X)$ is continuous and satisfies the relations

$$
s \circ h(0)=u_{1}, \quad s \circ h(1)=u_{2},
$$

and

$$
s \circ h(\tau) \in S(h(\tau)) \subset S, \quad \tau \in[0,1] .
$$

The proof is complete.

\section{REFERENCES}

[1] A. Cernea, "Qualitative properties of solution sets to a class of nonconvex nonclosed integral inclusions," in Proceedings of the Centennial "G. Vrănceanu” and the Annual Meeting of the Faculty of Mathematics (Bucharest, 2000), vol. 49, no. 2, 2000, pp. 123-131.

[2] A. Cernea, "On the set of solutions of some nonconvex nonclosed hyperbolic differential inclusions," Czechoslovak Math. J., vol. 52(127), no. 1, pp. 215-224, 2002.

[3] A. Cernea, "On the solution set of a nonconvex nonclosed second order differential inclusion," Fixed Point Theory, vol. 8, no. 1, pp. 29-37, 2007.

[4] S. A. Marano and V. Staicu, "On the set of solutions to a class of nonconvex nonclosed differential inclusions," Acta Math. Hungar., vol. 76, no. 4, pp. 287-301, 1997.

[5] S. A. Marano, "Fixed points of multivalued contractions with nonclosed, nonconvex values," Atti Accad. Naz. Lincei Cl. Sci. Fis. Mat. Natur. Rend. Lincei (9) Mat. Appl., vol. 5, no. 3, pp. 203-212, 1994.

[6] A. Petruşel, Operatorial inclusions. House of the Book of Science, Cluj-Napoca, 2002.

\section{Author's address}

\section{Aurelian Cernea}

Faculty of Mathematics and Informatics, University of Bucharest, Academiei 14, 010014 Bucharest, Romania

E-mail address: acernea@math.math.unibuc.ro 